\title{
Notas Farmacológicas ¿Cisapride en el banquillo de los acusados?
}

\section{Introducción}

Desde la llegada del cisapride al mercado se han detectado 270 casos de arritmias cardíacas severas asociadas a su administración (resultando fatales en 70 de estos casos). Las arritmias reportadas incluyen la taquicardia y fibrilación ventriculares, torsión de punta y prolongación del QT. El 23 de marzo del corriente la FDA (aegncia reguladora de medicamentos de EE.UU.) comunica un nuevo programa que promueve el uso limitado del cisapride. En Argentina, la ANMAT (Administración Nacional de Medicamentos, Alimentos y Tecnología médica) ha venido desarrollando medidas reguladoras progresivas con respecto a este fármaco. En noviembre del 1998 han modificado el prospecto y en febrero del 2000 han efectuado advertencias al cuerpo médico.

Es importante destacar que el $85 \%$ de las arritmias ocurrieron en pacientes con factores de riesgo identificables; y que durante los últimos doce años se han prescripto más de 190 millones de tratamientos en 90 países. Los EE.UU, Canadá y los países europeos decidieron continuar la comercialización del producto con algunas limitaciones. Estos son todos indicios de que la adecuada administración de la droga puede aún ser una herramienta terapéutica valiosa en la que los beneficios pueden superar a los riesgos. ${ }^{1-2}$ A fin de asegurar el uso racional del cisapride, la ANMAT ha dispuesto:

1) cambiar la condición de expendio del producto a venta bajo receta archivada, 2) ampliar la información ya existente en los prospectos,

3) limitar las indicaciones al reflujo gastroesofágico que no responde a las medidas habituales.

¿Qué precauciones debemos tener para prescribirlo adecuadamente? Para continuar prescribiendo adecuadamente este fármaco debemos memorizar las siguientes características farmacológicas:

\section{Contraindicaciones: ¿A quienes NO podemos darle cisapride?}

Pacientes que toman antibióticos macrólidos (Claritromicina, eritromicina y troleandomicina); algunos antifúngicos (fluconazol, itraconazol, y ketoconazol); inhibidores de la proteasa (indinavir y ritonavir); fenotiazinas (proclorperazina o prometazina); antiarrítmicos clase IA y III (quinidina, procainamida, sotalol, amiodarona); antidepresivos tricíclicos (como amitriptilina); otros antidepresivos (nefazodona y maprotilina); algunos antipsicóti$\cos$ (sertindol); y otros agentes (bepridil, sparfloxacina y jugo de uva).? No se debe utilizar en pacientes en quienes el aumento de la motilidad intestinal podría ponerlos en peligro ej.: hemorragia gastrointestinal, obstrucción mecánica o perforación.

Debe ser evitado en pacientes con historia de arritmias; electrocardiograma anormal; enfermedad pulmonar, renal o cardíaca; y condiciones que resulten en alteraciones electrolíticas, como uso de diurěticos perdedores de potasio y administración aguda de insulina.

Evitar su uso en pacientes con hipersensibilidad conocida o intolerancia a la droga. ${ }^{3}$

\section{Interacciones:}

El cisapride es metabolizado principalmente por la enzima citocromo P450 $3 \mathrm{~A}^{4}$. Las drogas que inhiben dicha enzima producen un aumento sérico del cisapride por lo cual se potencian los efectos adversos, como prolongación del QT (antifúngicos, macrólidos, etc ver contraindicaciones).

La administración conjunta con drogas anticolinérgicas podría comprometer los efectos beneficiosos del cisapride.

La aceleración del vaciado gástrico puede afectar la absorción de algunas drogas, por lo cual se deberá monitorear especialmente a los pacientes con drogas que poseen un rango terapéutico pequeño como teofilina, antiepilépticos, digitálicos etc. La ranitidina y cimetidina se absorben más rápida- mente con el agregado de cisapride.

En pacientes que reciben anticoagulantes orales se debe monitorear el tiempo de coagulación luego de unos días de comenzado el tratamiento con cisapride y luego de la suspención del mismo. ${ }^{3}$

\section{Precauciones:}

Se debe evaluar el riesgo- beneficio de su administración a pacientes con enfermedades asociadas a prolongación del QT, ya sea por el sindrome del QT prolongado, alteraciones electrolíticas o por drogas.

Se debe advertir a los pacientes que el cisapride puede acelerar los efectos sedativos de las benzodiazepinas y del alcohol.

Embarazo: se podría usar en este período sólo si el potencial beneficio justifica el riesgo para el feto.

Ancianos: se observa una moderada prolongación de la vida media de eliminación.

Efectos Adversos más frecuentes (> $1 \%$ de los pacientes):

Gastrointestinales: diarrea, dolor abdominal, constipación, dispepsia. Psiquiátricos: insomnio, ansiedad, nerviosismo. Otros: cefalea, rinitis, mareos, dolor precordial y de espalda, fatiga, depresión, deshidratación, mialgias, polaquiuria, vaginitis, trastornos de la visión y arritmias. ${ }^{3}$

\section{Mecanismo de acción y efectos farmacológicos:}

El cisapride estimula a la acetilcolina del plexo mesentérico. Es agonista de los receptores 5-HT4 serotoninérgicos, lo que resultaría en un aumento de la motilidad gastrointestinal y de la frecuencia cardíaca.

A nivel esofágico dosis de 4 a 10 mg IV aumentan la presión del esfínter esofágico inferior (PEEI) y la peristalsis esofágica inferior comparado a placebo y/o metoclopramida. En pacientes con reflujo gastroesofágico (RGE) y PEEI $<10 \mathrm{mmHg}$, el cisapride aumenta la fuerza de peristalsis y duplica la PEEI, en forma dosis dependiente. A nivel gástrico, dosis de $10 \mathrm{mg}$ IV u oral - $10 \mathrm{mg}$ oral 3 veces por día por más de 6 semanas, acelera el vaciado gástrico de líquidos y sólidos. Estudios clínicos mostraron que el cisapride puede reducir la pirosis nocturna asociada al RGE. Dos estudios controlados, uno usando una dosis de $10 \mathrm{mg} 4$ veces por día y el otro usando dosis de 10 y 20 mg 4 veces por día, mostraron mejoría en los síntomas de pirosis nocturna. No hubo mejoría en los síntomas diurnos, de regurgitación ni cambios histopatológicos en el esófago.3

\section{Indicación:}

Su úso se restringe al tratamiento sintomático de pacientes con ardor retroesternal nocturno debido a enfermedad por RGE, con poca respuesta a terapia habitual.

Comentario: Es muy importante extremar las precauciones para la prescripción de la droga ya que su utilización masiva y por distintos motivos es muy común en nuestro medio. Se debe utilizar únicamente en pacientes que padecen reflujo gastroesofágico y que no responden a las terapias habituales, es decir las medidas no farmacológicas, antiácidos o antiH2. ${ }^{4}$ Recordemos las medidas no farmacológicas habitualmente recomendadas: elevar la cabecera de la cama, distribuir la alimentación diaria en 4 comidas y 2 colaciones de escasa cantidad, evitar picantes, café, grasas, tabaco, cítricos, etc. Los antiácidos con hidróxido de magnesio y/o aluminio se usan en los pacientes con acidez nocturna, los antiH2 se usan en aquellos con síntomas más persistentes. En casos de RGE complicado (esófago de Barret, estenosis de EEI) en los que tienen esofagitis endoscópica erosiva, en los que no respondieron a los bloqueantes $\mathrm{H} 2$ se debe indicar inhibidores de la bomba de protones. Hasta el momento, no existen drogas proquinéticas alternativas que puedan reemplazar al cisapride para su principal indicación.

\section{Dra. Gabriela Lewin - Dra. Marcela Botargues}

Unidad de Medicina Familiar y Preventiva. Hospital Italiano de Buenos Aires.

Marcas comerciales que contienen cisapride en Argentina: MONODROGA: Cinacol (Gador); Cisap (Dominguez); Cisapride Ilab (Inmunolab); Cisapride (Klonal); Claminex (Bagó); Digenormotil (Baliarda); Digericon (Bristol); Etacril (Beta); Fisiodig (Casasco); Kinetizine (Cetus); Prepulsid (Janssen); Prepulsid Efervescente (Janssen); Pulsar (Phoenix); Pulsar Forte (Phoenix); Pulsar Reflux (Phoenix); Regalisa (Microsules Arg.). ASOCIACIONES: Digenormotil Plus (Baliarda); Fisiodig AG (Casasco); Gastril AG (Searle); Gastril Anti Gas Masticable (Searle); Gastrimet (Raffo); Gastrimet Enzimático (Raffo); Pulsar Enzimático (Phoenix) y Pulsar Plus (Phoenix) (fuente ANMAT)

Bibliografia

1. Nuevas Medidas en relación a la droga Cisapride. ANMAT previene. Comunicado de prensa. 28 de marzo de 2000.

2. Henney JE. From the Food and Drug Administration. JAMA 2000; 283(9): 1131

3. Center for Drug Evaluation and Research, FDA. Guidance for Industry. Labeling Guidance for Cisapride tablets. Internet: http://www.fda.gov/cder/guidance/index.htm.

4. Mc Ouaid KR. Gastroesophageal Reflux Disease. Current Medical Diagnosis \& Treatment 1999. Ed. 38 th. Appleton \& Lange. 564-8

Centros de información

Para obtener información complementaria o para realizar el reporte correspondiente acerca de alqún caso relacionado con esta droga en Argentina, se ruega comunicarse con:

* Red Provincial de Vigilancia Farmacéutica: Coordinador de la RPVF: TE/FAX 0221 4290952, e-mail: rpvf@colfarma.com.ar

* Laboratorio de Control de Calidad TE/FAX 0221 4290952/75, e-mail: labfarma@infovia.com.ar

* Sistema Nacional de farmacovigilancia de la ANMAT: TELEFAX: (011) 4340-0866; Email: snfvg@anmat.gov.ar 